\title{
Study on DCT Power Shift Control Simulation for Electric Drive System
}

\author{
Xianghuan Liu ${ }^{1,2,}{ }^{*}$, Yubo Xiao ${ }^{2}$, Junxing Gong ${ }^{2}$, Jun $\mathrm{Ma}^{2}$, Xuming $\mathrm{Pi}^{2}$ \\ ${ }^{1}$ College of Mechanical and Electrical Engineering, Central South University, Changsha, Hunan \\ 410083 China; \\ 2Zhuzhou Gear Co., Ltd., Zhuzhou, Hunan 412000 China. \\ *Corresponding author email:0512655@163.com
}

Keywords: Electric vehicles; DCT; AT; uninterrupted power.

\begin{abstract}
A two-speed dual clutch transmission (DCT) model was built, while a Clutch-To-Clutch shift control algorithm based on the closed loop of input speed was designed following the slipping torque control theory. On the mat lab/Simulink platform, models were completed for vehicle, DCT gearbox transmission chain and shift control software. A simulation was carried out with a blade electric SUV to verify the effectiveness of power shift control logic and the theoretical feasibility of speed closed-loop shift control algorithm and reveal the relationship between shift time and slipping friction work. The results prove that the shift logic and speed closed-loop shift control algorithm designed in this paper can effectively control the switching of dual clutches in the power shift process to guarantee the steady and controllable power output.
\end{abstract}

\section{Introduction}

Currently, most electric vehicles utilize one-speed reducer, which significantly restricts the climbing performance, acceleration performance and maximum speed of vehicles, so the application of gearbox in electric vehicles becomes a hot topic in the research. At present, the common gearbox for electric vehicles in the market is two-speed automated manual transmission (AMT) gearbox, which can greatly resolve the problems of low-speed climbing and maximum speed for vehicles. However, AMT gearbox is defective for its prominent power interruption and poor drivability.

Using the wet dual clutch structure in the two-speed DCT gearbox independently developed by our company for electric drive system, this paper designs a power shift control strategy and presents a simulation study to verify its effect.

\section{Analysis of Structure and Physical Properties}

The DCT structure studied in this paper is presented in Fig. 1. In this gearbox, a pair of wet clutches is used in place of synchronizer in AMT gearbox. In this gearbox, the 1st speed and 2nd speed are provided with a wet clutch and an independent hydraulic control system each, so their shift can be controlled freely. The wet clutches can transmit the maximum torque of $400 \mathrm{Nm}$, so power shift can be achieved through their slipping control.

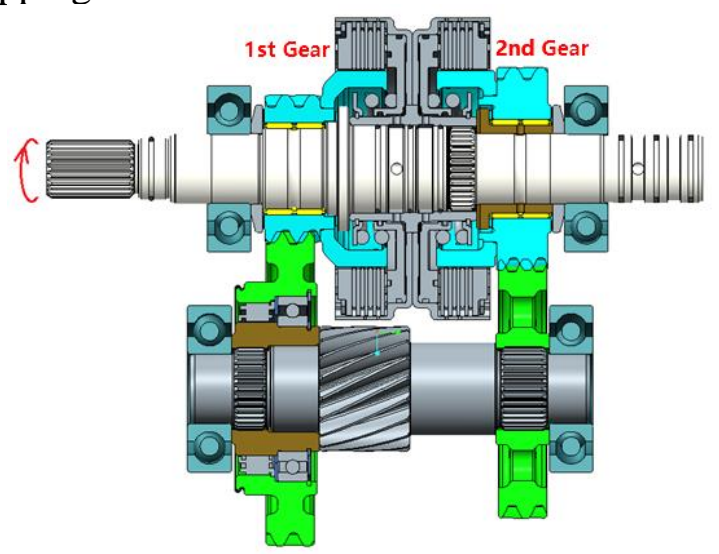

Fig 1. Wet two-speed DCT structure 


\subsection{Characteristic Test of Static Friction Torque Transmission for Wet Clutches}

To find out the static friction torque transmission characteristics of wet clutches, a test bench for slipping torque was constructed as shown in Fig. 2.

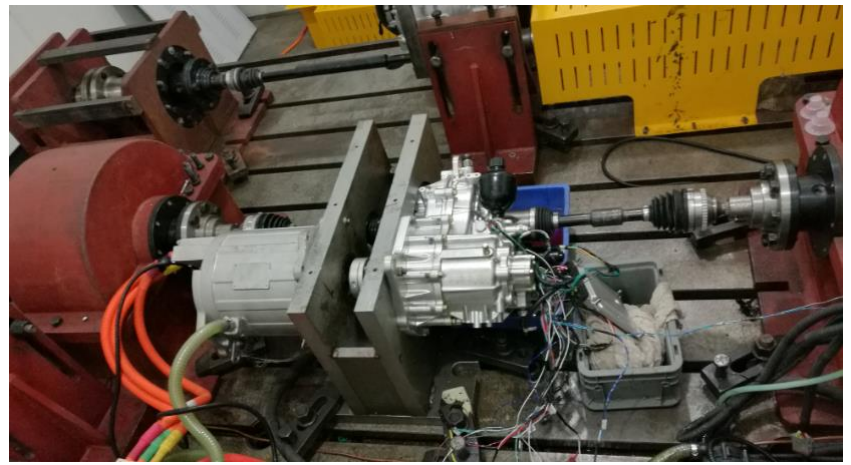

Fig 2. Test bench of slipping torque

The test was conducted in the following way: stabilize the pressure of clutch to a certain value, gradually increase the input torque of gearbox, test the input speed and output speed of gearbox, and confirm whether they match with the transmission ratio. When the torque increases to a critical level, the clutch will slip to cause the mismatch between input shaft speed and output shaft speed. The critical torque is the maximum static friction torque under the pressure.

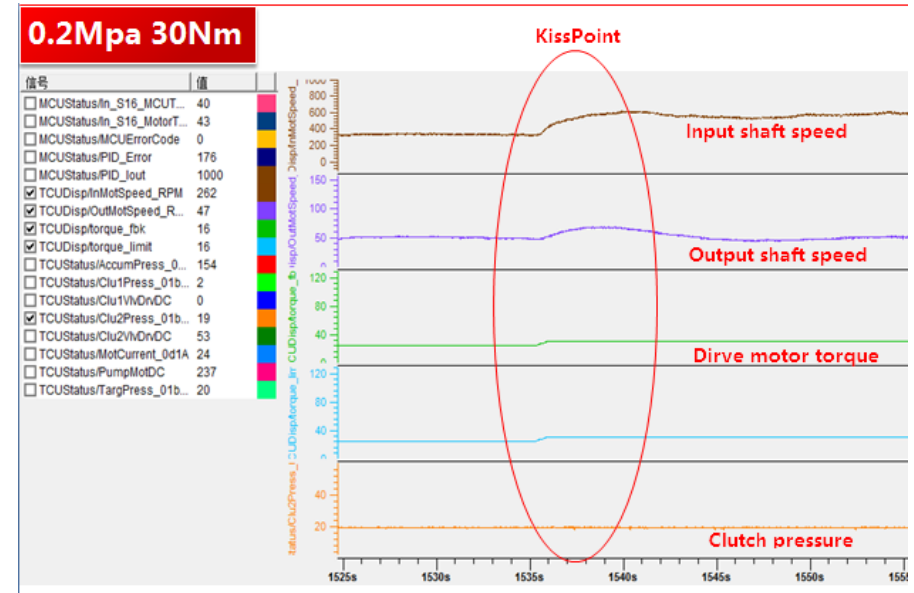

Fig 3. Capture of static friction torque extremes

After testing different pressure points, the correlation curve between static friction torque extremes and pressure is presented in Fig. 4.

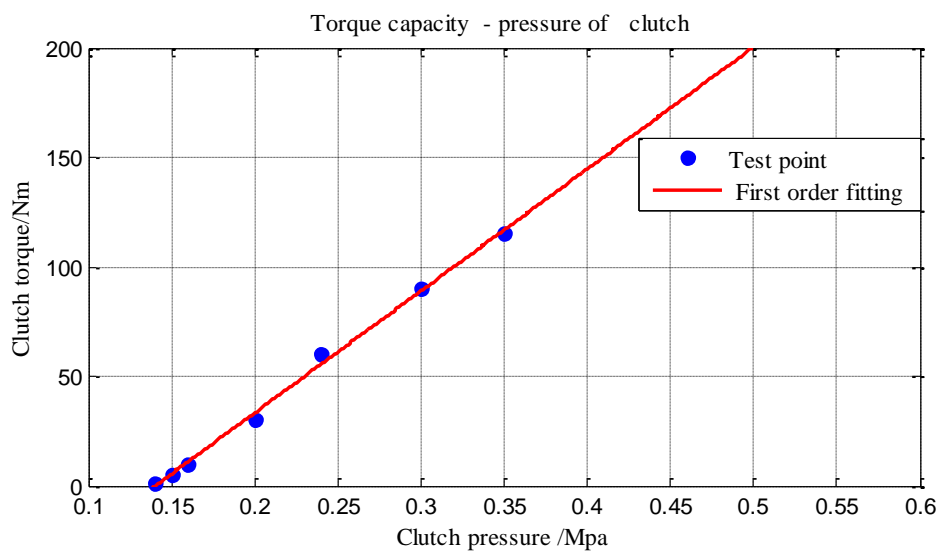

Fig 4. Static friction torque capacity of clutch under different pressures 


\section{Control Software Modeling and Virtual Vehicle Modeling}

The simulation system designed in this paper consists of control software model and virtual vehicle model, which are coupled into a model in the loop (MIL) system. The virtual vehicle model provides the environment for the logic verification and control performance verification of the control model. The effect of the MIL system is shown in Fig. 5.
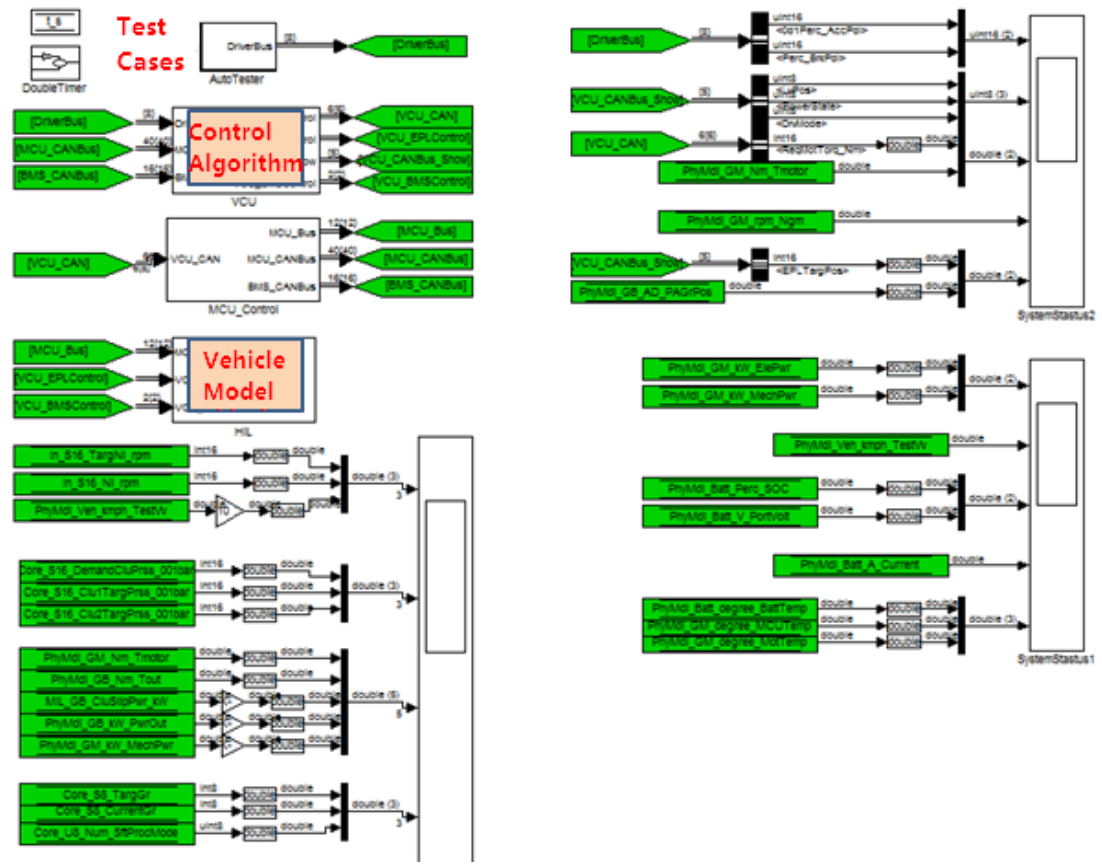

Fig 5. The effect of the MIL system is shown

\subsection{Virtual Vehicle Model.}

The architecture of virtual vehicle model is presented in Fig. 6. Its key idea is that the torque of power system acts on the vehicle mass model through transmission device to generate the acceleration, which is integrated to obtain the current speed, and fed back to the power system inversely through transmission chain, so as to form a stable closed-loop system. Fig. 7 presents the dual clutch slipping torque transmission model. If inputting different pressures and speeds, the model will give different output torques. Apart from calculation of output torque, this model can also calculate the slipping friction work, so it can be used to compare the slipping friction work in the shift process when different control parameters are used. The formula for calculation of slipping friction work is as follows:

$$
W_{f}=\int_{0}^{t}|T \Delta \omega| d t
$$

This paper particularly describes the control algorithm, but the virtual vehicle model is not elaborated.

In Fig. 7, Tin is input torque, T_c1 is 1st speed clutch transmission torque, T_c2 is 2nd speed clutch transmission torque, and Tout is output torque. Nain is input speed, N_c1 is 1st speed clutch driven speed, N_c2 is 2nd speed clutch driven speed, and Knout is output speed. Moreover, i1 is 1st speed transmission ratio, while i2 is 2 nd speed transmission ratio.

The 1st speed gear pair and 2nd speed gear pair are both fixed transmission ratio, so there is:

$$
\begin{aligned}
& \text { N_1 }=\text { N_out } \times \text { i1 } \\
& \text { N_2 }=\text { N_out } \times \text { i2 }
\end{aligned}
$$

In the proposed design of gearbox in this paper, i1 is approximately twice of i2, so N_1 is nearly twice of N_2 as well. The drive speeds of both clutches are Nain, so only one clutch can be closed synchronously during movement. If two clutches are closed simultaneously, either clutch may certainly slip and even seize. 


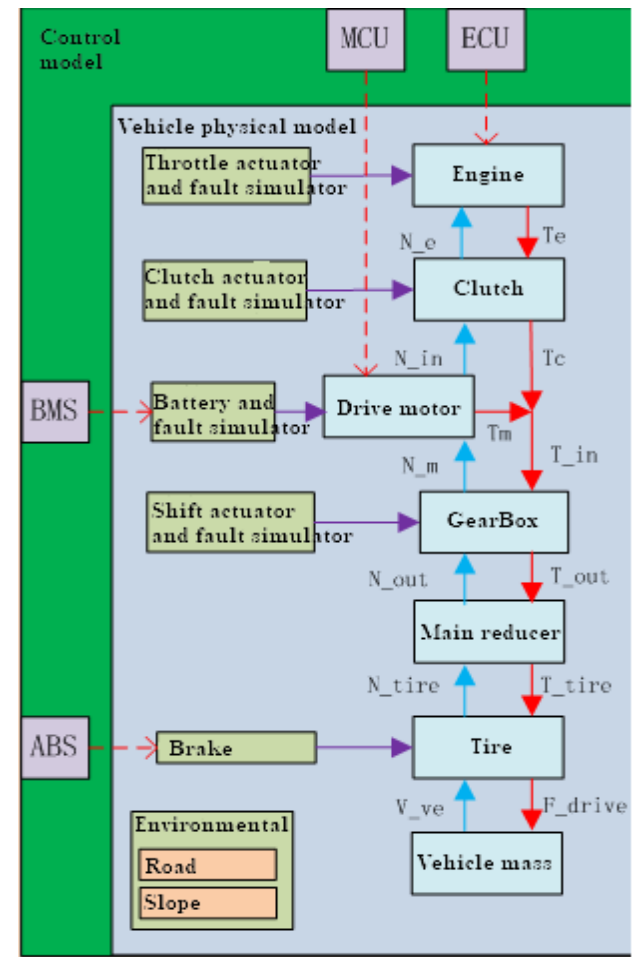

Fig 6. Architecture of virtual model simulation model

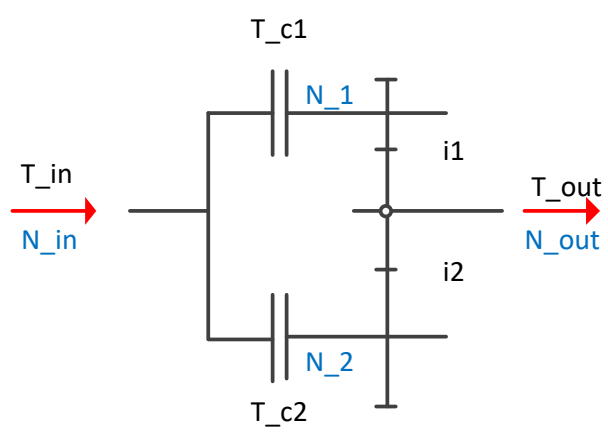

Fig 7. 2DCT transmission principles

When the 1st speed clutch is closed synchronously, and the 2nd speed clutch is opened:

$$
\text { Tout }=\text { T_c1 } \times \text { i } 1=T \_ \text {in } \times \text { i1 }
$$

When the 1st speed clutch is closed synchronously, and the 2nd speed clutch slips:

$$
\begin{gathered}
\text { Tout }=\mathrm{T}_{-} \mathrm{c} 1 \times \mathrm{i} 1+\mathrm{T} \_\mathrm{c} 2 \times \mathrm{i} 2 \\
\mathrm{~T} \_\mathrm{c} 1=\mathrm{Tin}-\mathrm{T} \_\mathrm{c} 2 \\
\mathrm{~T} \_\mathrm{c} 2=\mathrm{f}(\mathrm{Pc} 1, \triangle \mathrm{n}, \mathrm{Temp})
\end{gathered}
$$

When the 1st speed clutch slips, and the 2nd speed clutch slips:

$$
\begin{aligned}
& \text { Tout }=\mathrm{T}_{-} \mathrm{c} 1 \times \mathrm{i} 1+\mathrm{T} \_\mathrm{c} 2 \times \mathrm{i} 2 \\
& \mathrm{~T} \_\mathrm{c} 1=\mathrm{f}(\mathrm{Pc} 1, \triangle \mathrm{n}, \mathrm{Temp}) \\
& \mathrm{T} \_\mathrm{c} 2=\mathrm{f}(\mathrm{Pc} 1, \Delta \mathrm{n}, \mathrm{Temp})
\end{aligned}
$$


When the 1 st speed clutch is opened, and the 2nd speed clutch is closed synchronously:

$$
\text { Tout }=\text { T_c } 2 \times i 2=T \_ \text {in } \times \text { i2 }
$$

When the 1st speed clutch slips, and the 2nd speed 1clutch is closed synchronously:

$$
\begin{gathered}
\text { Tout }=\text { T_c1 } \times \text { i1 } 1+\mathrm{T} \_\mathrm{c} 2 \times \mathrm{i} 2 \\
\mathrm{~T} \_\mathrm{c} 1=\mathrm{f}(\mathrm{Pc} 1, \Delta \mathrm{n}, \mathrm{Temp}) \\
\text { T_c2 }=\text { Tin - T_c1 }
\end{gathered}
$$

$\mathrm{F}(\mathrm{Pc} 1, \triangle \mathrm{n}, \mathrm{Temp})$ stands for the function of the correlation of slipping torque with acting pressure, speed difference and temperature. It can be measured in the test.

On the basis of the above correlation between torque and speed, the model is built as shown in Fig. 8.
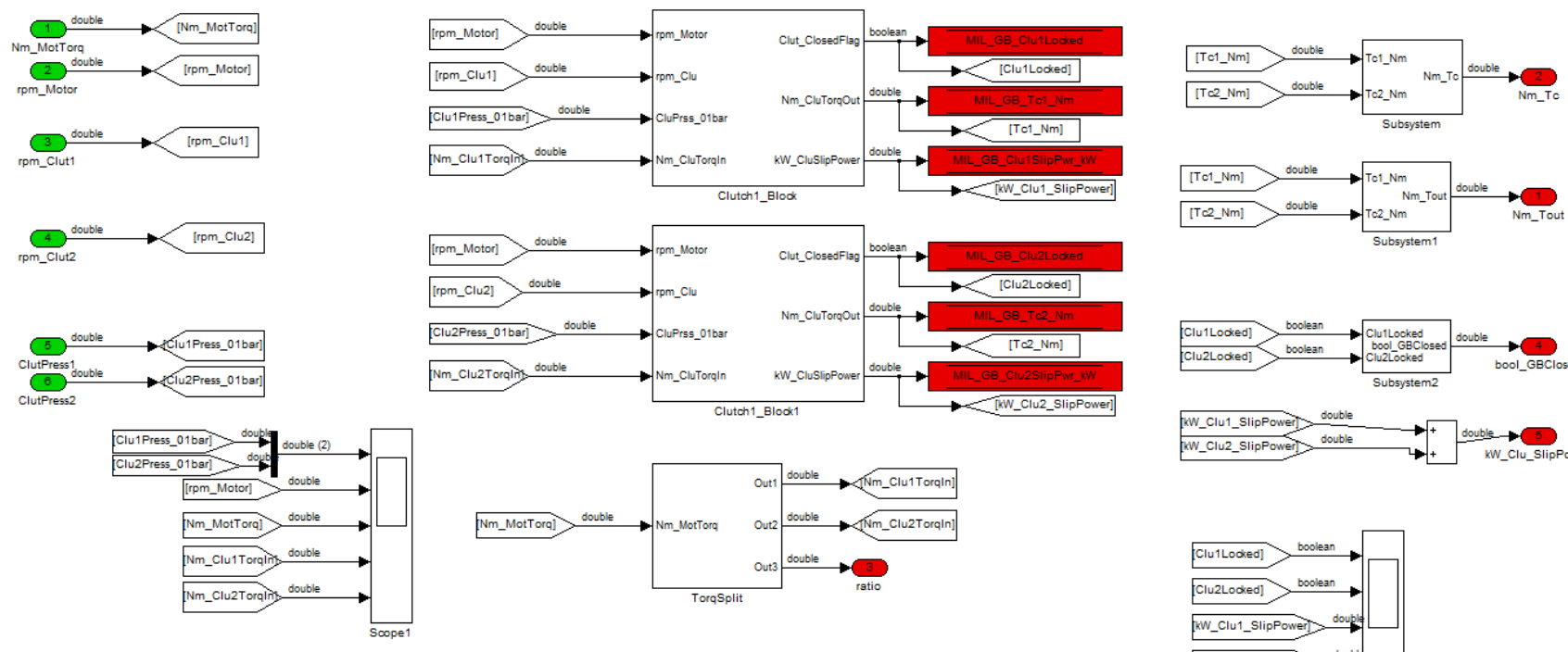

Fig 8. Dual clutch output torque characteristic model

\subsection{Shift Logic.}

Fig. 9 presents a sketch of shift logic designed in this paper. There are two shift routes, i.e. up-shift process at the top and down-shift process at the bottom.

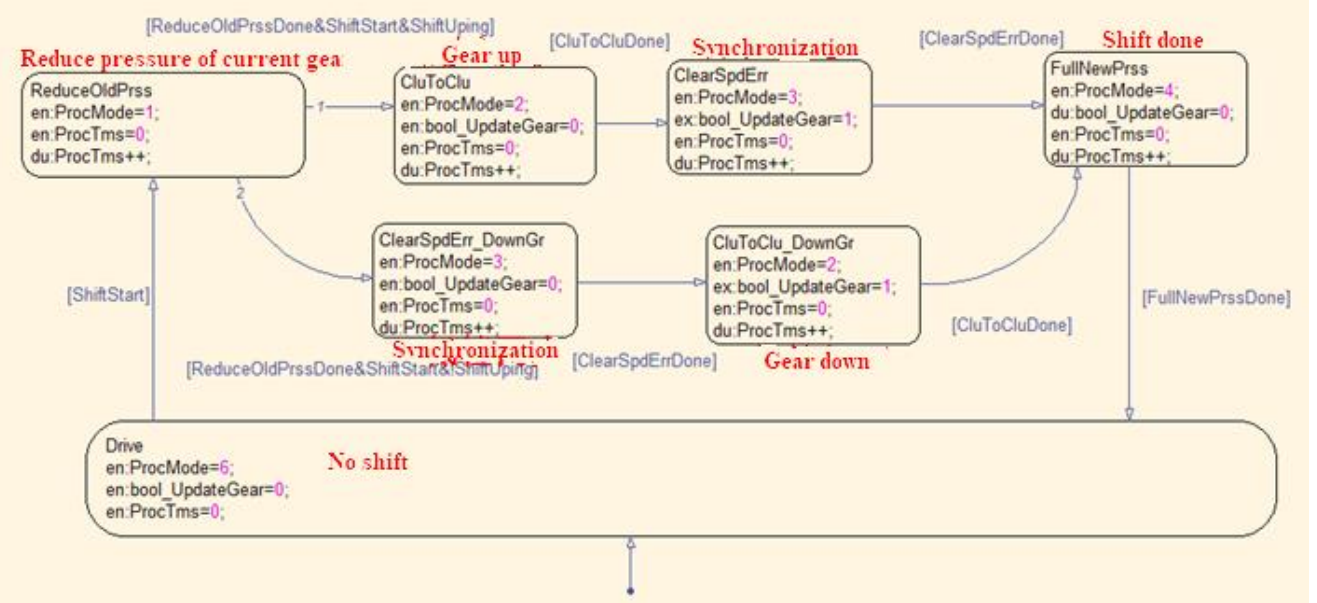

Fig 9. Sketch of shift control status 
Up-shift Process: Old speed (Clu1) pressure decrease - high speed pressure increases - high speed synchronization - new speed (Clu2) full pressure;

In the process, old speed (Clu1) pressure decrease can remove the extra acting force on the old speed clutch. The target pressure can be found in the curve in Fig. 4 on the basis of actual input torque. At this time, the rate of pressure relief does not affect the shift performance, so the higher, the better.

High speed pressure increase can build up the pressure of high speed (2nd speed) clutch. Equations 2.5 and 2.6 can be used to obtain the torque of gearbox. At this time, the high-speed clutch is slipping. Along with the increase of high speed clutch pressure, the slipping torque increases as well, while the torque transmitted by low speed clutch decreases gradually, and its pressure goes down as well. When the input torque of gearbox is shifted to the high-speed clutch, the low speed clutch can release all of its pressure, so high-speed pressure increase is completed. It must be noted that the low speed clutch should be controlled to prevent slipping in the process.

During high speed synchronization control, the main goal is to control the decrease track of input shaft speed. The input speed can be modulated in two ways:

1). The high-speed clutch pressure remains unchanged, i.e. output torque is not changed, but the input motor torque is properly lowered, so the motor can automatically decelerate to get close to synchronization. This way is featured by lower slipping friction work, but it still requires restoring the torque of motor and building up the pressure after synchronization, and it recovers the power slowly;

2). The input motor torque is not changed, but the clutch pressure is adjusted to regulate the input speed. When the torque of motor is unchanged, higher clutch pressure leads to quicker decrease of motor speed. This way can recover the power rapidly without changing the output power, but it causes higher slipping friction work.

In this paper, the torque of motor is not changed, but pressure is regulated to control the progress of synchronization. The specific control algorithm is detailed in the next section.

New speed (Clu2) full pressure means to increase the new speed clutch pressure to full pressure, which can prevent the slipping of clutch during maximum output torque. As the clutch has been synchronized before, pressure increase will not affect the drivability in this process. Therefore, the faster, the better.

Down-shift Process: Old speed (Clu2) pressure decrease - low speed synchronization-low speed pressure increases - new speed (Clu1) full pressure;

In this process, old speed (Clu2) pressure decrease and new speed (Clu1) full pressure are similar to those in the up-shift process, so they are not detailed again.

The differences between down-shift and up-shift are as follows: synchronization control is conducted before low speed pressure increase in the down-shift process. At this time, the low speed clutch has a negative speed difference. If low speed pressure increase happens, negative torque will be generated. The higher pressure, the larger negative torque. In this case, there will not be any output of positive driving power before low speed synchronization.

In the low speed synchronization, the goal is also to control the input speed of gearbox (i.e. motor speed in the structure proposed in this paper). In this paper, the control is achieved by properly lowering the high-speed clutch pressure, so the motor runs at a controllable over speed, which increases to the speed for 1 st speed synchronization. If the acceleration does not satisfy the requirements, the torque of motor is further adjusted to accelerate.

In the low speed pressure increase process, the control aims to stabilize the speed of motor. As the low speed synchronization is completed, low speed pressure increase will not generate negative torque, and the low speed clutch pressure can be increased gradually, while the high-speed clutch pressure is released at the same time.

\subsection{Speed Track Synchronization Control Algorithm.}

The speed track in the synchronization process reflects the output torque smoothness and slipping friction work of clutch, so it is crucial to control the synchronization track of input speed. In this paper, the high-speed clutch pressure is regulated to lower the input speed in the up-shift process, 
while the high-speed clutch pressure is modulated to increase the input speed as well in the downshift process. Hence, the key to the control of speed track is the control of high speed clutch pressure.

Fig. 10 presents a determination method designed in this paper for pressure control. On the basis of theoretically needed pressure, pressure correction is added to control the system pressure. Among them, the theoretical needed pressure is the pressure needed to transmit the current torque, while pressure correction is the pressure automatically generated for regulation considering the progress of speed synchronization, and mainly used to adjust the synchronization track of speed.

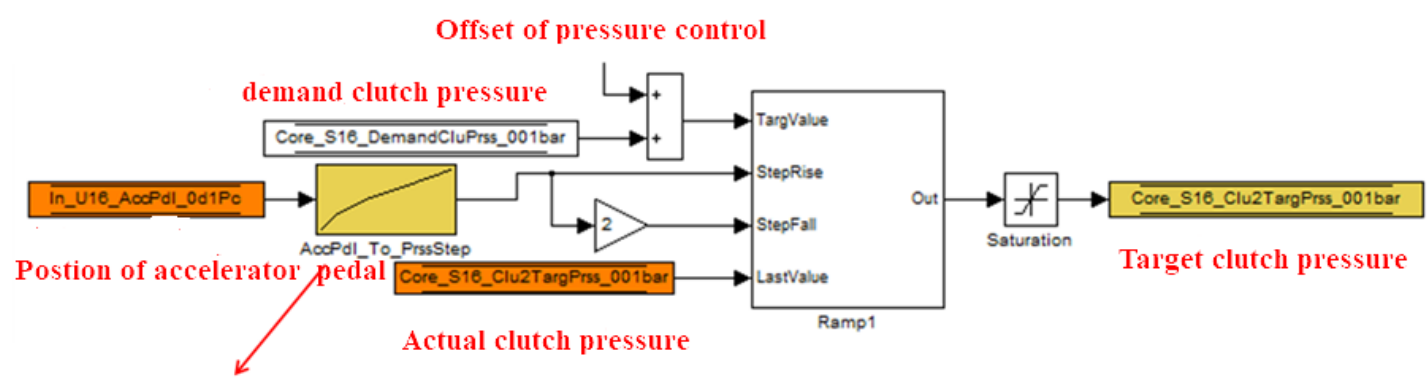

Table of target pressure change slope

Fig 10. Determination process of updated target pressure

The determination process of pressure correction is shown in Fig. 11 and follows the idea that the error between actual speed and target speed is used to find a speed for reducing the error. At different errors, different target speed gradients are calibrated to control the synchronization speed track. After the target speed gradient is obtained, it is taken as the goal in the PI control calculation to obtain the pressure correction. The PI control algorithm is shown in Fig. 12.
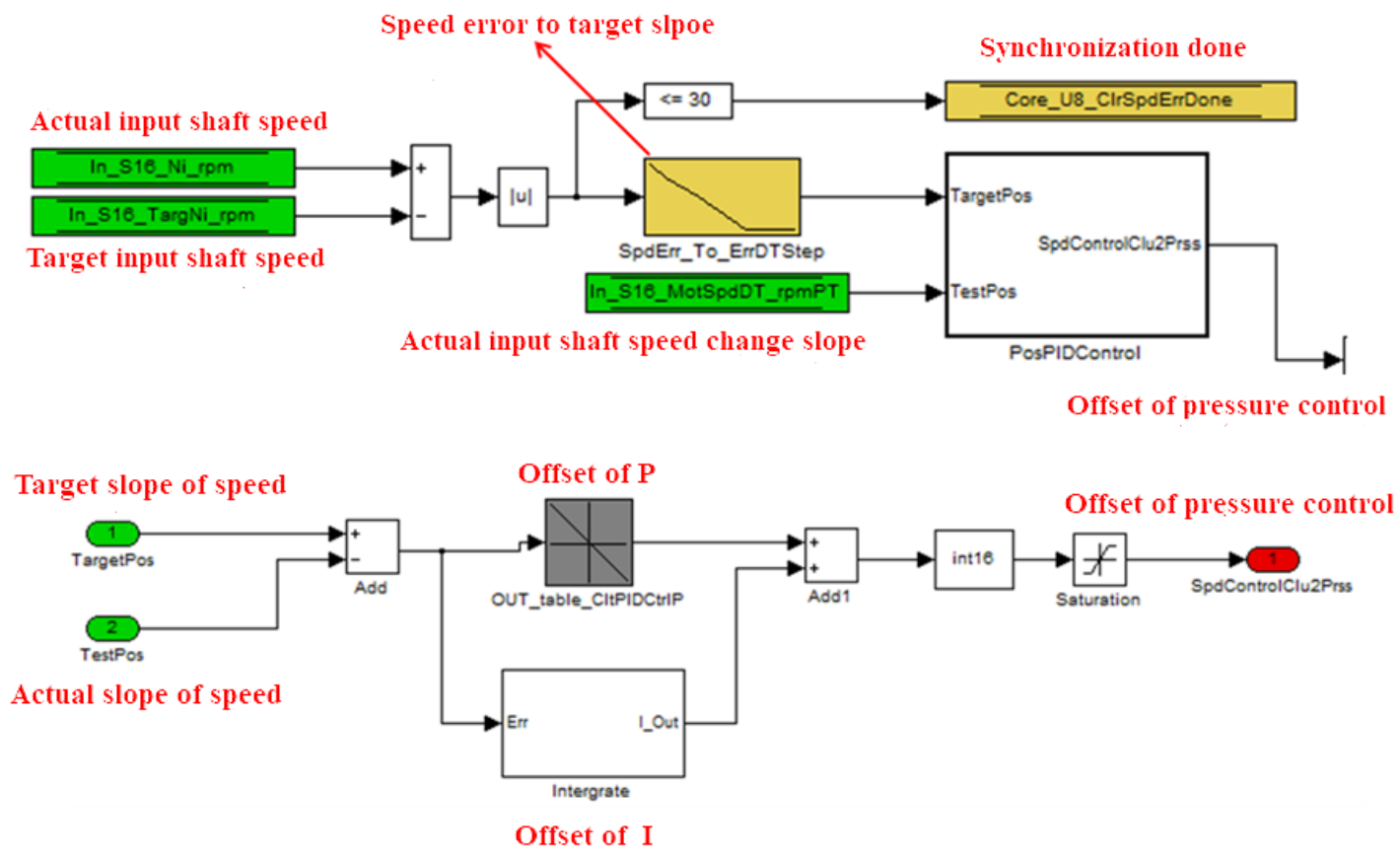

Fig 11. PI algorithm for target speed gradient control

\section{Simulation Analysis}

This paper designs the operating conditions for simulation as follows: a vehicle keeps a speed, its motor maintains the maximum output torque, and its gearbox repeats the control of power shift from 1 st speed to 2 nd speed and from 2 nd speed to 1 st speed. The speed of motor is 5,000rpm before upshift, and 2,500rpm after up-shift. It is reversed in the down-shift process. Simulation is carried out 
to verify the shift control process and the effectiveness of synchronization algorithm, as well as the influence of synchronization rate on slipping friction work.

\subsection{Up-shift Control Simulation.}

The effect of dynamic up-shift control is presented in Fig. 12. After analyzing the actual speed and target speed curves of motor, it is found that motor speed is controlled very steadily without any over speed or shake. When the speed is about to synchronize, the actual speed grows close to the target speed as shown in Fig. 13, which is caused by calibrating different target gradients at different errors, and very beneficial to the comfort of shift. Moreover, it can avoid the abrupt change of torque when the clutch changes from dynamic friction to static friction. In Fig. 12, the clutch pressure curves of high and low speeds switch steadily as well without abnormal shake. After comparing motor power, DCT output power, and slipping friction work curve, it is revealed that power loss is inevitable under full power due to slipping friction work, as the high speed of motor is featured by constant power.

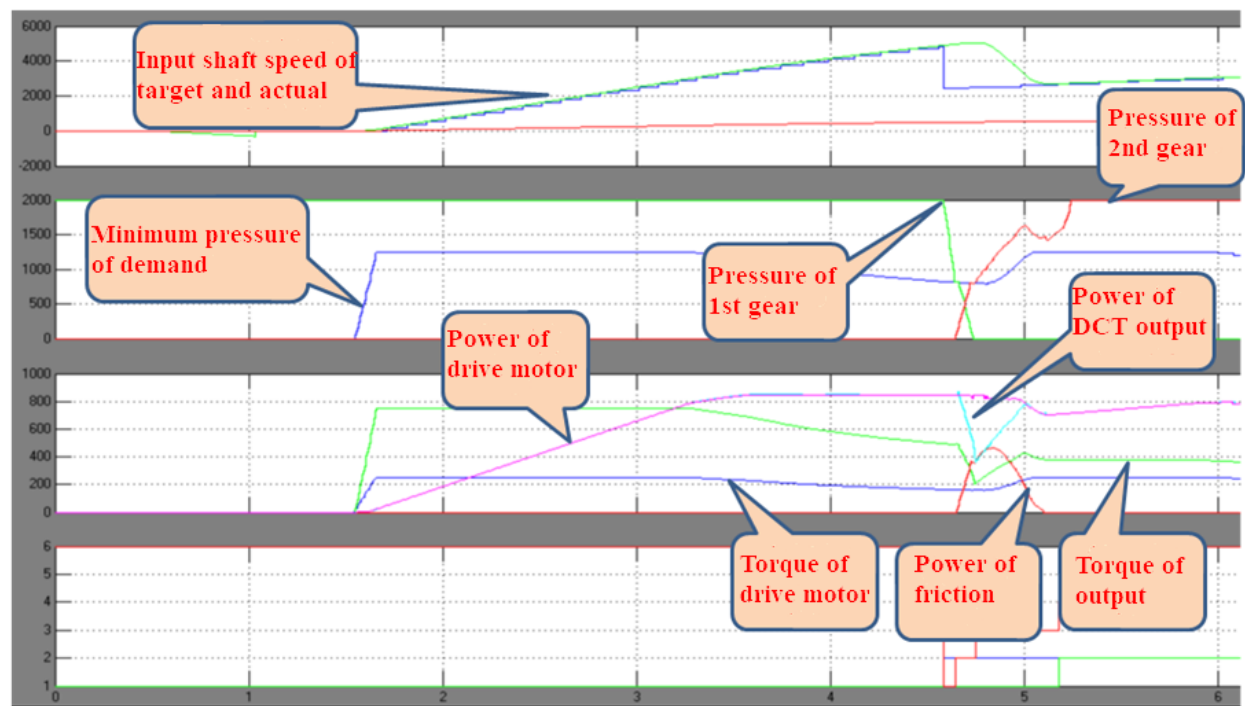

Fig 12. Simulation effect of dynamic up-shift control

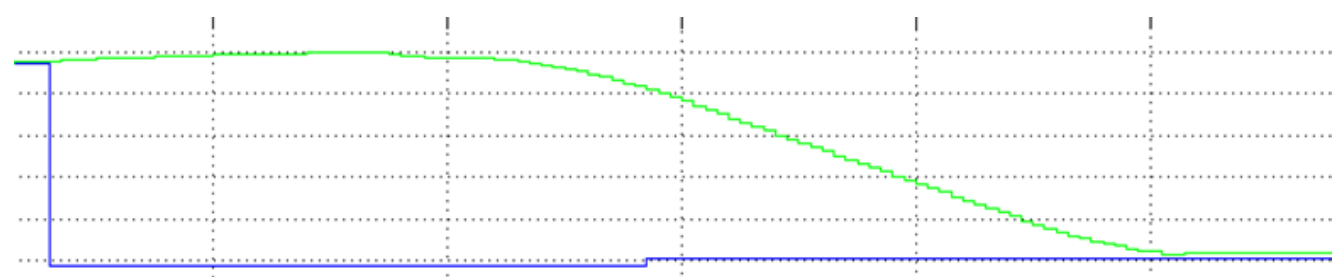

Fig 13. Synchronization speed approximation curve

The statistical result of slipping friction work is $12.76 \mathrm{~kJ}$ as shown in Fig. 14 .

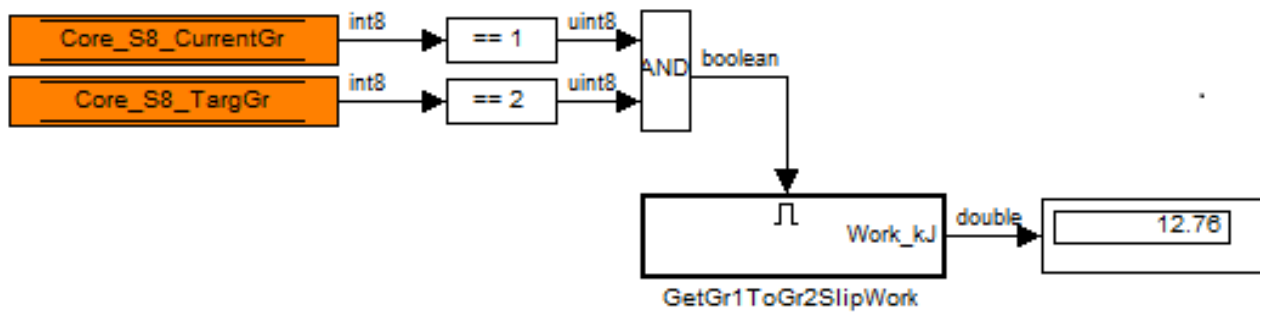

Fig 14. Statistics of dynamic up-shift control slipping friction work

\subsection{Down-shift Control Simulation.}

The effect of dynamic down-shift control is shown in Fig. 15. The control of motor speed is stable, while the clutch pressure curves of high speed and low speed are switched very steadily. Similarly, 
motor power, DCT output power and slipping friction work curves are compared to show the inevitable power loss under full power due to slipping friction work. The statistical result of slipping friction work is $5.671 \mathrm{~kJ}$ as shown in Fig. 16.

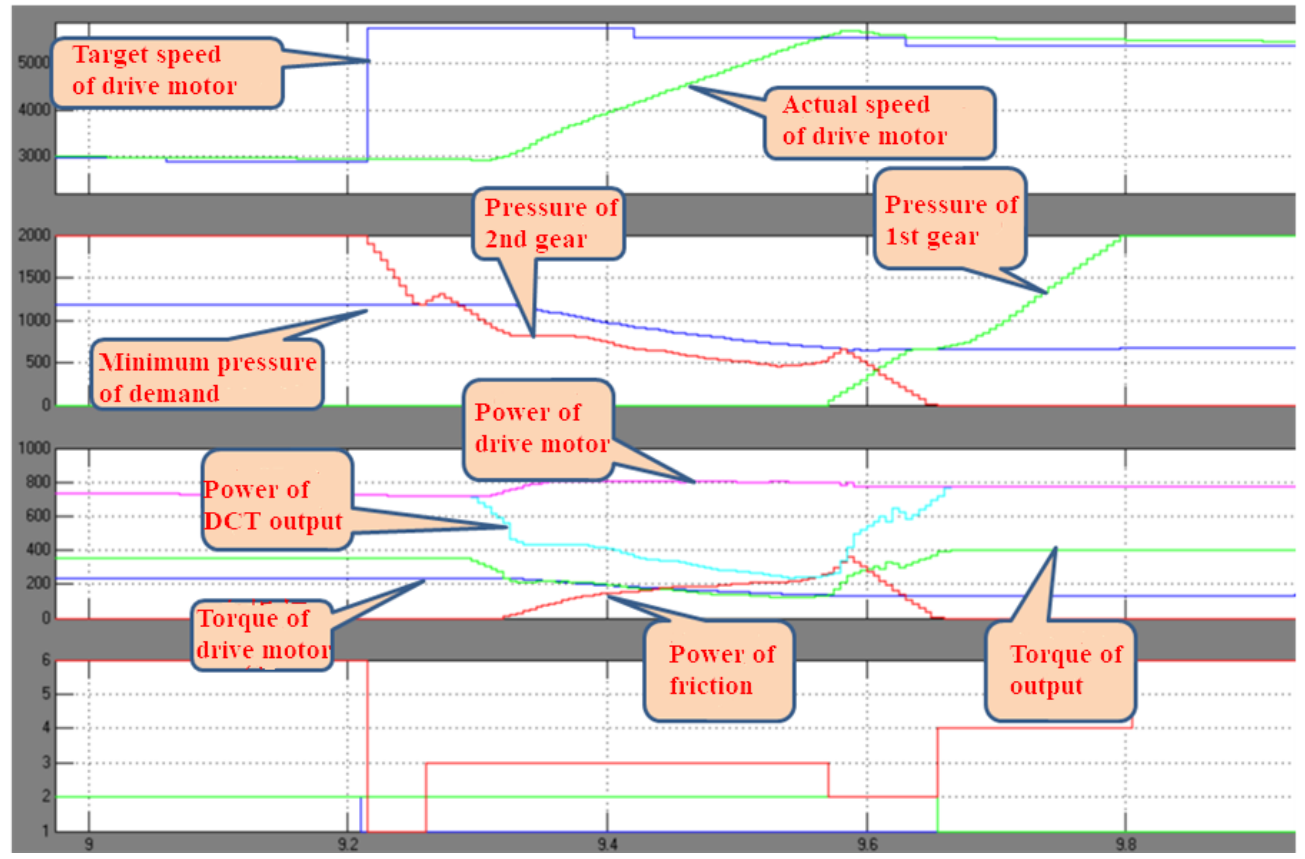

Fig 15. Simulation effect of dynamic down-shift control

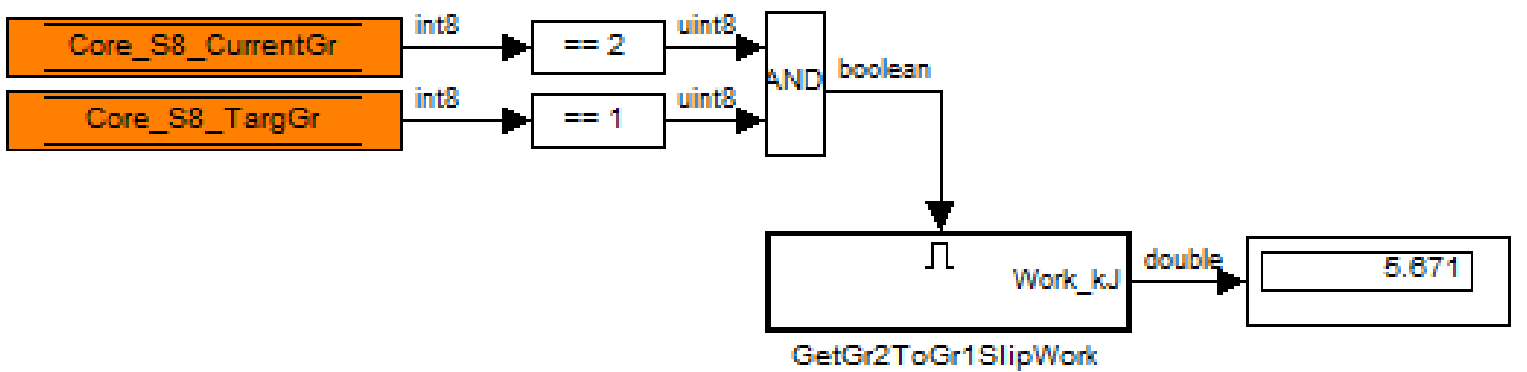

Fig 16. Statistics of dynamic down-shift control slipping friction work

\subsection{Influence of Synchronization Rate on Slipping Friction Work.}

The analysis is carried out as follows: by calibrating the correlation between speed error and target speed gradient in Fig. 1, the synchronization at different rates is achieved. Data are obtained from simulation and stored for comparison to find out the difference between the clutch slipping friction works of the motor under the same input power at the stage of synchronization.

Table 1. Power at the stage of synchronization.

\begin{tabular}{|c|c|c|}
\hline \multicolumn{3}{|c|}{1 st Speed to 2nd Speed } \\
\hline No. & Synchronization time & Slipping friction work \\
\hline 1 & $250 \mathrm{~ms}$ & $11.14 \mathrm{~kJ}$ \\
\hline 2 & $350 \mathrm{~ms}$ & $12.76 \mathrm{~kJ}$ \\
\hline 3 & $600 \mathrm{~ms}$ & $17.86 \mathrm{~kJ}$ \\
\hline 4 & $1100 \mathrm{~ms}$ & $33.18 \mathrm{~kJ}$ \\
\hline
\end{tabular}




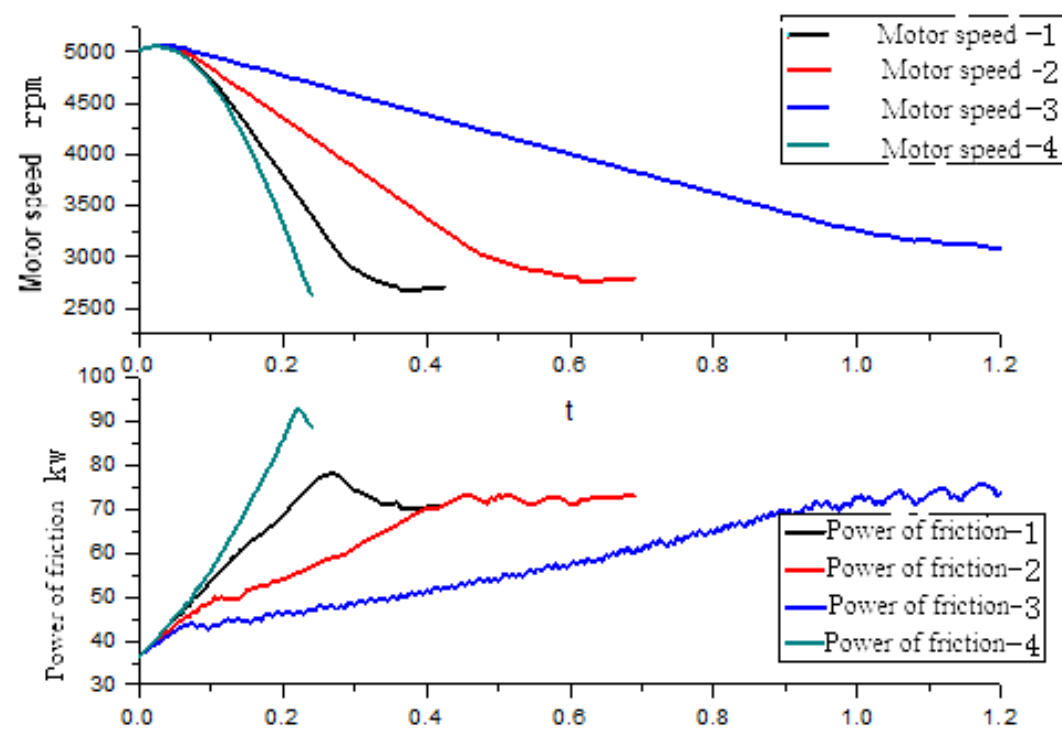

Fig 17. Curve comparison at different synchronization rates during up-shift

Table 2. As verified in the simulation calculation

\begin{tabular}{|c|c|c|}
\hline \multicolumn{3}{|c|}{ 2nd Speed to 1st Speed } \\
\hline No. & Synchronization time & Slipping friction work \\
\hline 1 & $200 \mathrm{~ms}$ & $2.39 \mathrm{~kJ}$ \\
\hline 2 & $300 \mathrm{~ms}$ & $5.671 \mathrm{~kJ}$ \\
\hline 3 & $500 \mathrm{~ms}$ & $14.14 \mathrm{~kJ}$ \\
\hline 4 & $1000 \mathrm{~ms}$ & $22.1 \mathrm{~kJ}$ \\
\hline
\end{tabular}

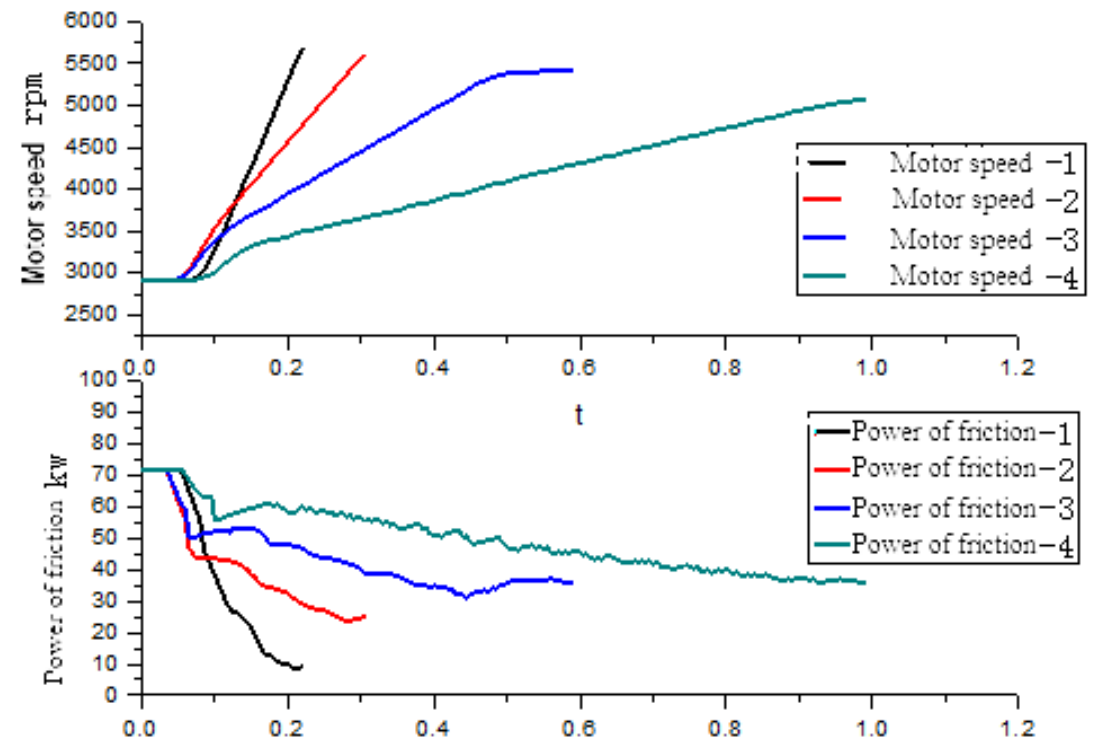

Fig 18. Curve comparison at different synchronization rates during down-shift

\section{Conclusion}

As verified in the simulation calculation, the virtual vehicle model designed in this paper can effectively reflect the characteristics of electric vehicle and its DCT gearbox and provide a test platform for the development of DCT control software. This paper designs a power shift control process and synchronization speed track closed-loop control algorithm. After simulation test for verification, the control effect meets the expectation, while the power output control is smooth and can be calibrated and adjusted according to the needs of vehicle. Moreover, this paper compares the influence of different synchronization time on the slipping friction of clutch. The shorter 
synchronization time, the lower slipping friction work. In the practical vehicle calibration, the synchronization time should be shortened as much as possible, but attention should be also paid to the drivability of vehicle.

\section{References}

[1]. Han Peng, Chen Xiusheng, Li Xingzhong, et al. A Study on the Shift Schedule of DCT Based on Integrated Powertrain Control [J]. Automotive Engineering, 2015, 5: 538-543.

[2]. Gao Song, Yuan Yean, Chen Man, et al. Study on Engine Speed Control in DCT Slipping Progress [J]. Chinese Journal of Automotive Engineering Chin J Auto Eng., 2016, 6: 397-403.

[3]. Sun Dongle, Wang Cong. Control Strategy of DCT Shift Based on Engine with Negative Torque [J]. China Mechanical Engineering, 2013, 19: 2692-2697.

[4]. Luo Wei. Intelligent shift method in DCT system based on genetic neural networks [J]. Manufacturing Automation, 2013, 19: 68-71,102.

[5]. Fan Jansen, Wu Tong Feng, Jin Gooding. Analysis of Structure Layout of Electric Drive System of EV [J]. Machinery, 2003, 11: 38-40.

[6]. Zoo Bo, Tan Kun, Yang Gang, et al. Research on Distributed Multi-wheel Electric-drive System Design for a Special Vehicle [J]. Vehicle \& Power Technology, 2017, 4: 1-6. 